\title{
SAGES guidelines for the management of hiatal hernia
}

\author{
Jeffrey H. Peters
}

Received: 20 August 2013/ Accepted: 30 August 2013/Published online: 3 October 2013

(C) Springer Science+Business Media New York 2013

Among the principles of current health care reform is to promote better value. Although "value" is not a term most of us are used to including in our everyday clinical practices, its components-quality and cost—are. Achieving higher quality at lower cost is a win-win circumstance that few would not embrace if given the opportunity. Although much has been written about the relative quality of our much-maligned health care system, the truth is, it is actually already high. That being said, it is also clear that there is much room for improvement by raising clinical quality across the spectrum of health care settings and lowering costs whenever possible. Guidelines can help make this possible. They are not meant as rigid dictates to be applied to every patient. Good clinical care is much too nuanced for that. Rather, guidelines provide a blueprint upon which to start and standards to reduce variability. It is in the former that quality can be improved, and the latter that provides an opportunity for cost reduction.

This brings us to the SAGES "Guidelines for the Management of Hiatal Hernia," which appears in this issue of Surgical Endoscopy [1]. This document is orders of magnitude better than surgical guidelines produced in decades past. It represents an enormous effort on the part of a number of individuals who carefully evaluated and critiqued published evidence. They have utilized the GRADE system, which is widely recognized as the highest standard methodology for guideline development. The document is comprehensive and clear; it provides 28 separate recommendations encompassing a clinical spectrum of the management of hiatal hernia, from diagnosis to surgical technique and perioperative management. Considerations

J. H. Peters $(\bowtie)$

University of Rochester, Rochester, NY, USA

e-mail: jeffrey_peters@urmc.rochester.edu in the pediatric and bariatric populations have been included. There is no doubt that this document will provide useful guidance to the health care community for years to come.

It is important to emphasize that this is a guideline for the management for hiatal hernia, not gastroesophageal reflux disease. They overlap but are different. This important fact should perhaps be better emphasized in the preamble to the guidelines. The guideline is divided into sections ranging from diagnosis to postoperative management. The section on diagnosis is generally excellent, although the statement that "expert opinion suggests that a contrast swallow showing normal motility may replace the need for catheter-based manometry study in patients with paraesophageal hiatal hernia" is a bit overstated, given the relatively poor standard to which most barium studies of the esophageus are performed and interpreted. The guidelines comment that "heartburn and reflux symptoms are uncommon with paraesophageal hernias." In fact, heartburn and regurgitation are among the most common symptoms in patients with paraesophageal hernia. Later in this section, the authors comment that vascular compromise may cause anemia. In fact, vascular compromise is neither necessary nor present in most patients with anemia; it is often the result of mechanical trauma at the level of the diaphragm, so-called Cameron ulcers, or occult erosions, both generally in the presence of normal mucosal blood supply. The section on natural history is good and well outlines improvements in our understanding of the natural history of paraesophageal hernia over the years. The inclusion of sections on repair during bariatric operations and management in the pediatric population is excellent and attests to the comprehensive nature of the guideline.

The section on predictors of outcome highlights postoperative nausea and vomiting, age, obesity, and hiatal 
surface area. Although each of these has been reported as a potential factor affecting outcome, the data remain sparse. As such, the guideline limits recommendations to treat postoperative nausea and vomiting "aggressively," although defining this term would have been helpful.

Among the most important aspects of the guideline ate those that relate to technical considerations, and here I will comment on specific recommendations. Discussion of laparoscopic versus open repair is appropriate and accurate. Guideline 10, recommending excision of hernia sac, is likely overstated; there is little evidence that this affects outcome in any way. The pros and cons of prosthetic reinforced repair are among the most controversial aspects of the repair of both sliding and paraesophageal hernias. The guideline provides an excellent review of the issue and appropriate recommendations. Guidelines 11 and 12 could be combined into a single recommendation. Guideline 13 recommendations are based on "weak" evidence that a fundoplication must be performed during repair of a sliding hiatal hernia to address reflux. Historical experience and reasonable evidence clearly show that hernia repair alone is an insufficient treatment for a patient with sliding hiatal hernia and reflux. The text goes on to cite evidence limited to paraesophageal hernias, ignoring data on sliding hiatal hernias. Although fundoplication may be optional in some patients with paraesophageal hernia, particularly in urgent or emergent circumstances, the standard of care is to include fundoplication in the repair of sliding hernias. Admittedly, few if any prospective randomized trials have compared repair with and without fundoplication; nevertheless, the historical evolution of the standard of care should be acknowledged here.
Guideline 14 comments on tailoring the fundoplication, a topic of interest for some 20-30 years. The guidelines limit this discussion to consideration only with respect to manometric findings. As stated, the guidelines are likely true, although the question is a bit incomplete. The authors do not review the literature on other pertinent preoperative risk factors for postoperative dysphagia, including the presence of dysphagia before the operation and measures of bolus transport. In fact, the presence of dysphagia before surgery is the single most important risk factor for dysphagia after surgery. These two important factors should also be acknowledged in the tailoring question. The discussions of a potential short esophagus and postoperative management are excellent, and Guidelines 15-19 are appropriate. Guideline 22 might more reasonably state that both laparoscopic or open revisional surgery are appropriate and that the causes of the failure should be sought.

In the end, however, the SAGES guidelines committee and the authors have produced a thoughtful and useful document to inform surgeons undertaking the management of patients with hiatal hernia. These guidelines will no doubt serve as a base upon which future evidence can be included-and, importantly, also inform investigators of areas in need of further study.

Disclosures Dr. Peters has no conflict of interest or financial ties to disclose.

\section{Reference}

1. SAGES. Guidelines for the management of hiatal hernia. Surg Endosc (in press) 